\title{
Overview of Pathogens causing Nosocomial Infections in Various Wards of a Tertiary Health Care Hospital and their Antimicrobial Susceptibility Pattern - A Prospective Surveillance Study
}

\author{
D. Saikeerthana* (D), P. Prabha, V. Vijayashree and Gogula N.M. Krishna \\ Department of Microbiology, Government Medical College and ESI Hospital, \\ Coimbatore - 641 015, Tamilnadu, India.
}

\begin{abstract}
The major problem encountered in recent days due to the advent of emerging antimicrobial resistance strains is Hospital Acquired Infections (HAIs). The spread of HAl is mainly through the hospital staff and the hospital settings' inanimate surfaces. The study includes the surveillance of hospital surfaces, including wards and ICUs, by taking swabs dipped in normal saline. Pseudomonas aeruginosa (53.8\%) was found to be the most common pathogen isolated, followed by Klebsiella species (18\%), MethicillinSensitive Staphylococcus aureus (16\%), Coagulase-Negative Staphylococci (6\%), Methicillin-Resistant Staphylococcus aureus (3\%). The widespread presence of bacterial sensitivity to antimicrobials and the modifications insensitivity forms the basis for designing antibacterial therapy's practical recommendations and rational use of antimicrobials. Proper display of all Information, Education, and Communication (IEC) materials at appropriate places play a significant role in preventing nosocomial infections. Audiovisual aids and training to the staff play utmost importance in preventing the spread of HAls. All these can reduce the occurrence and outbreak of nosocomial conditions. Overall, these minimize health care costs.
\end{abstract}

Keywords: Hospital Acquired Infections, Pseudomonas aeruginosa, Information, Education and Communication, Klebsiella species, antimicrobial resistance

*Correspondence: keerthanaanush@gmail.com; +91 9965576295

(Received: February 10, 2021; accepted: August 25, 2021)

Citation: Saikeerthana D, Prabha P, Vijayashree V, Krishna GNM. Overview of Pathogens causing Nosocomial Infections in Various Wards of a Tertiary Health Care Hospital and their Antimicrobial Susceptibility Pattern-A Prospective Surveillance Study. J Pure Appl Microbiol. 2021;15(4):1940-1946. doi: 10.22207/JPAM.15.4.15

(c) The Author(s) 2021. Open Access. This article is distributed under the terms of the Creative Commons Attribution 4.0 International License which permits unrestricted use, sharing, distribution, and reproduction in any medium, provided you give appropriate credit to the original author(s) and the source, provide a link to the Creative Commons license, and indicate if changes were made. 


\section{INTRODUCTION}

Hospital Acquired Infections (HAls) serves as one of the major threat in the medical field even after the beginning of much antimicrobial therapy and progress in supportive care management. ${ }^{1}$ The hospital environment, objects/instruments, and health-care workers are likely to get populated by various microbial agents. Disease transmission leads to morbidity and mortality due to contact with things handled by infected and colonized patients and direct communication with such patients ${ }^{1,2}$ and also at least 90,000 of them die due to HAI. ${ }^{3,4}$ Healthcare-associated infections are the fifth major cause of death in critical-care hospitals. The proportion of HAl patients in developing countries is neither recorded nor appropriately reported due to various reasons like difficulty in collecting reliable data and improper follow-up of HAI surveillance system. ${ }^{5} \mathrm{~A}$ summary of evidence has estimated the frequency of HAls to be $7.6 \%$ in high-income countries and $10.1 \%$ in middleincome countries. ${ }^{6}$

These HAls produce mortality at high rates in neonates, especially in premature and low birth weight babies. Worldwide, out of the five million neonatal deaths, $4 \%$ to $56 \%$ of neonatal deaths are due to this hospital-acquired infections. ${ }^{7}$ Moreover, these HAI occur mainly in developing countries; there is the fact that about $98 \%$ of these neonatal deaths occur in developing nations. In high-income countries, the prevalence of $\mathrm{HAl}$ is about $7.6 \%$ which is less than $10.1 \%$ in middle-income countries. ${ }^{6,10}$ Multidrug-resistant pathogens are now emerging as a global threat, and neonates are most commonly affected by their low immune status. Most frequently, the contaminated hands of the health care workers and the hospital environmental surfaces serve as the primary source of infection for all these HAls. Methicillin-Resistant Staphylococcus aureus (MRSA) strains continue to be the main threat to community and hospital-acquired diseases. These staphylococcus shows high resistance to the drug Vancomycin, one of the most common susceptible drugs for Staphylococcus aureus. ${ }^{8}$ In this study, about $17.5 \%$ were MRSA strains, and $5 \%$ of vancomycin-resistant strains were identified. ${ }^{8}$ Certain studies tell us the persistence of bacterial pathogens on the hospital floors and wards inanimate surfaces. These serve as the primary source of transmission of nosocomial pathogens and remain a danger to susceptible patients or health care workers. ${ }^{9}$

The present study aims to detect the potential source of infection responsible for $\mathrm{HAl}$ in various wards, labor rooms, ICUs and identification of bacterial isolates along with their antibiotic resistance pattern.

\section{MATERIALS AND METHODS}

A prospective surveillance study of pathogens causing a potential infection source at various tertiary care hospital wards was conducted. The study period was about four months, from May 2019 to August 2019. A total of 150 samples were obtained from sterile swabs dipped in normal saline. The samples were taken from the floors, walls, air conditioning vent, door handles, medicine racks, oxygen cylinders, bed, and cots. After collection, the swabs were immediately transferred to the laboratory and inoculated into the brain heart infusion broth for 24 hours. After incubation, the samples were sub-cultured in Blood agar and MacConkey agar. Thereafter, incubated at $37^{\circ} \mathrm{C}$ for the next 24-hour period. All positive cultures were identified using biochemical tests such as-indole, sugars, citrate, urease, and triple sugar iron medium. The disc diffusion method in Muller Hinton Agar (MHA) determined the antibiotic sensitivity profile of each identified organism, as per the Clinical and Laboratory Standards Institute (CLSI) 2019 guidelines. Resistance to Methicillin was detected on all Staphylococcus aureus isolates using Cefoxitin (30mcg).

\section{RESULTS}

Around 150 samples were collected from the medical, surgical, paediatric ICUs and wards, labor room, and post-operative ward. Among the samples, 43.3\% (65) were positive for bacterial isolates. Table 1 identifies possible sources of infection within various wards. In this, $93.3 \%$ of bacteria isolated from surgical wards, $73.3 \%$ from paediatric wards, $66.7 \%$ from medicine wards, $30 \%$ from labor room, and $20 \%$ from medical, surgical, and paediatric ICUs. Pseudomonas aeruginosa was the predominant organism isolated in major wards and ICUs, followed by Klebsiella species, Methicillin-Sensitive Staphylococcus aureus 
(MSSA), Coagulase-Negative Staphylococci (CONS), Methicillin-Resistant Staphylococcus aureus (MRSA). Apart from these, Enterococcus spp was also isolated rarely. Overall, the study revealed a predominant presence of strains of Pseudomonas aeruginosa from the medical, surgical, paediatric, work room and post-operative intensive care rooms from which the swabs were taken.

Among the 150 cultures done, 65 plates showed growth in the blood agar and MacConkey agar which was followed by biochemical tests as a further step to identify the pathogenic species. Among those 65 positive cultures, 53.8\% (35) were isolated as Pseudomonas aeruginosa, $18 \%(12)$ isolates were identified as Klebsiella pneumonia, $16 \%$ (11) isolates were MethicillinSensitive Staphylococcus aureus (MSSA), 6\% (4) were Coagulase-Negative Staphylococcus (CONS), 3\% (2) were Methicillin-Resistant Staphylococcus aureus (MRSA) and 1.6\% (1) were identified as Enterococcus species.

The antibiotic sensitivity testing for all the isolates was done using the Kirby-Bauer disc diffusion method as per standards to identify the multidrug-resistant strains (MDR) and MRSA. The various drugs used for Gram negative bacilli were ampicillin, piperacillin-tazobactam, ceftazidime, cefepime, cefaperazone-sulbactam, amikacin, gentamicin, ciprofloxacin, imipenem, meropenem and cotrimoxazole. For Gram positive isolates, amoxicillin-clavulanic acid, vancomycin, linezolid, cefoxitin, erythromycin, clindamycin and tetracyclines were used. The predominant isolate of this study was Pseudomonas aeruginosa. Among 65 positive cultures identified, 53.8\% (35) were positive for Pseudomonas. It was identified by using various biochemical tests such as oxidase

Table 1. Positive Bacterial growth in different areas of the hospital

\begin{tabular}{lcccc}
\hline No. & $\begin{array}{c}\text { Places where } \\
\text { swabs taken }\end{array}$ & $\begin{array}{c}\text { Number of } \\
\text { swabs taken }\end{array}$ & $\begin{array}{c}\text { Positive } \\
\text { culture }\end{array}$ & $\begin{array}{c}\text { Percentage } \\
\text { (\%) }\end{array}$ \\
\hline 1 & Medicine ICU & 20 & 4 & 20 \\
2 & Surgical ICU & 25 & 5 & 20 \\
3 & Paediatric ICU & 20 & 4 & 20 \\
4 & (NICU\&PICU) & & & \\
5 & Medicine Ward & 15 & 10 & 66.7 \\
6 & Surgical ward & 15 & 14 & 93.3 \\
7 & Paediatric ward & 15 & 11 & 73.3 \\
8 & Labour room & 20 & 6 & 30 \\
\hline
\end{tabular}

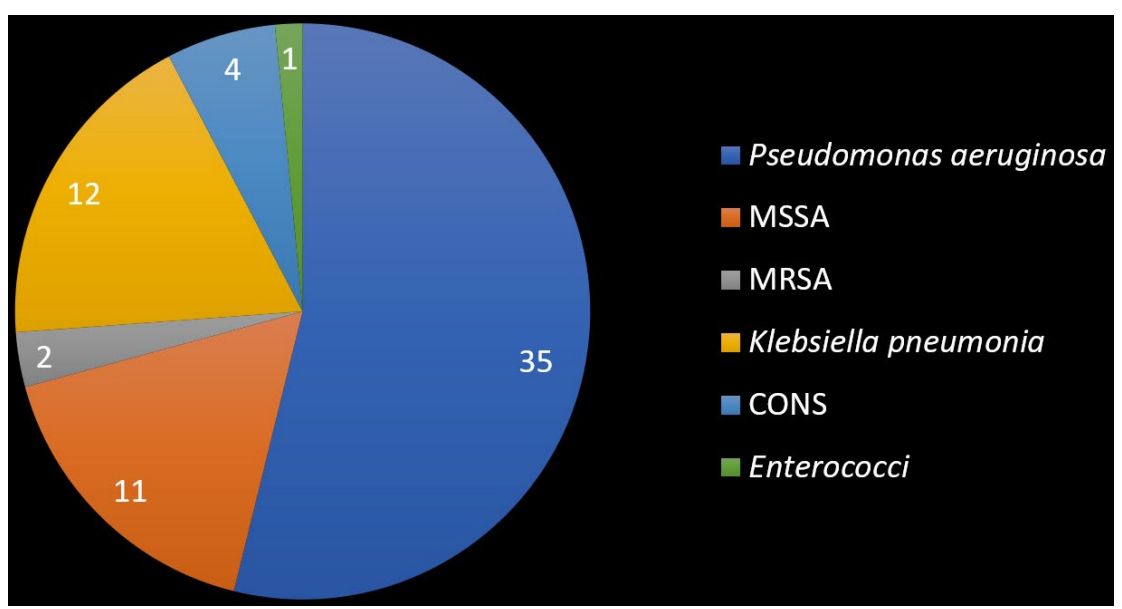

Fig 1. Pie diagram showing the number of isolates. 
positive, citrate positive, TSI (K/K-), indole negative and also with the help of pigment production.

Among 35 isolates of Pseudomonas aeruginosa identified, $74 \%$ (26) were sensitive to piperacillin-tazobactam, ceftazidime, cefepime, cefaperazone-sulbactam, amikacin, gentamicin, ciprofloxacin, imipenem, meropenem and 26\% (9) were identified as resistant to all the above drugs and sensitive only to higher group of drugs like colistin. All isolates of Pseudomonas aeruginosa identified were intrinsically resistant to Cotrimoxazole by disc diffusion method. Fig.
2 illustrates the sensitive strains and Multi drug resistant Pseudomonas aeruginosa strains.

Klebsiella pneumonia was the next predominate organism that was isolated. Among 65 (53.8\%) positive cultures, 12 (18\%) were identified as Klebsiella pneumonia by doing biochemical tests such as indole negative, citrate positive, Mannitol motility medium showing its fermentation of mannitol and also its non-motile behaviour. Klebsiella pneumonia was isolated from all wards except surgical ward.

Table 2. Distribution of Resistant strains and Methicillin Resistant Staphylococcus aureus (MRSA)

\begin{tabular}{lcccc}
\hline $\begin{array}{l}\text { Name of the } \\
\text { isolate }\end{array}$ & Ward /ICUS & $\begin{array}{c}\text { Number of } \\
\text { isolate }\end{array}$ & $\begin{array}{c}\text { Sensitive } \\
\text { strains }\end{array}$ & $\begin{array}{c}\text { Resistant } \\
\text { strains }\end{array}$ \\
\hline $\begin{array}{l}\text { Pseudomonas } \\
\text { aeruginosa }\end{array}$ & Surgical ward & 11 & 9 & 2 \\
& Medical ward & 4 & 3 & 1 \\
& Labour room & 4 & 3 & 1 \\
& Paediatric ward & 7 & 3 & 4 \\
& Post op & 4 & 3 & 1 \\
& PICU/NICU & 2 & 2 & 0 \\
Klebsiella & SurgicallCU & 2 & 2 & 0 \\
pneumonia & Medical ICU & 1 & 1 & 0 \\
& Surgical ward & 2 & 2 & 0 \\
& Medical ward & 3 & 2 & 1 \\
& Labour room & 2 & 1 & 1 \\
& Paediatric ward & 2 & 2 & 0 \\
MRSAs & Post op & 1 & 1 & 0 \\
& PICU/NICU & 1 & 0 & 1 \\
& Medical ICU & 1 & 1 & 0 \\
\hline
\end{tabular}

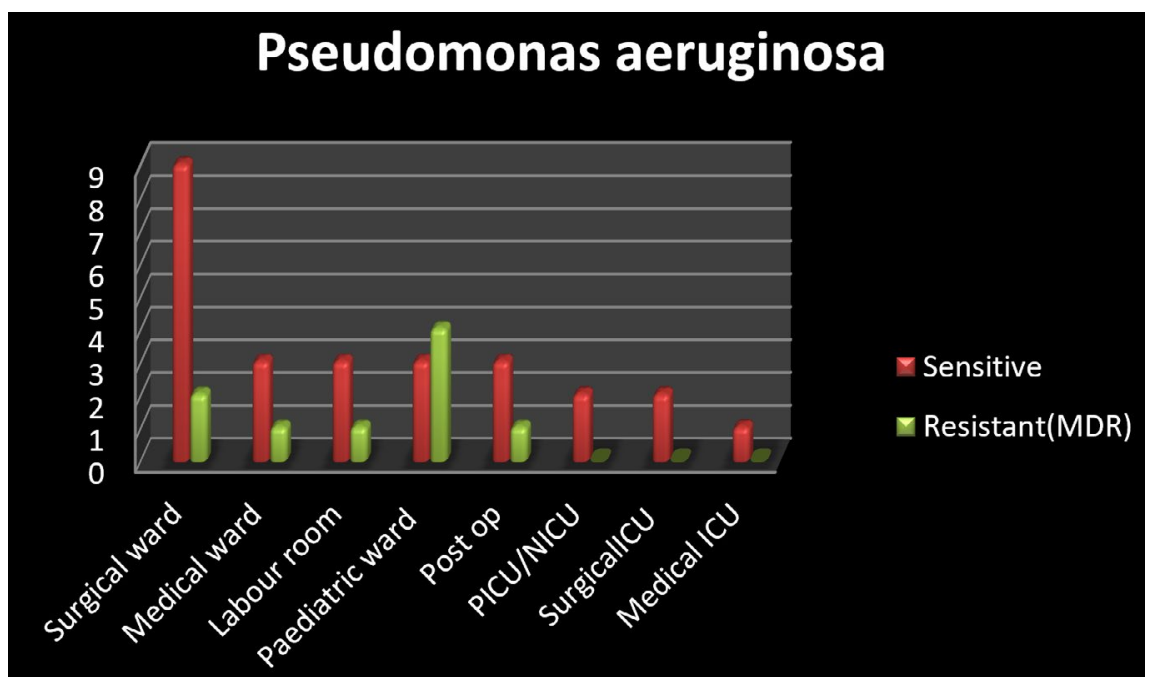

Fig 2. illustrates the sensitive strains and MDR Pseudomonas aeruginosa strains. 
Among twelve isolates, nine of them were sensitive to drugs like amoxicillin-clavulanic acid, cefotaxime, ceftrioxzone, ceftazidime, imipenem, amikacin, gentamicin and cotrimoxazole and three isolates were resistant to all the above drugs. Klebsiella pneumonia is intrinsically resistant to ampicillin. Fig. 3 illustrates the sensitive strains and resistant strains of Klebsiella pneumonia.

From sixty five positive cultures, seventeen (26\%) were identified as Gram positive cocci in clusters and only one (1.5\%) isolate was seen as Gram positive cocci in pairs. Staphylococcus aureus identified by doing positive catalase test, coagulase test and cefoxitin disc for identification of MSSA and MRSA. Only two isolates were found to be MRSA in postoperative wards. Enterococcus species was identified by positive bile esculin test and is sensitive to all drugs including high level gentamicin. Table 2 shows the distribution of Multi-drug resistant strains and MethicillinResistant Staphylococcus aureus (MRSA).

\section{DISCUSSION}

Infectious bacteria found in different parts of hospitals are often known to be the common cause of HAl. Infected patients, colonized with bacteria and healthcare providers are the main source of contamination. The main source of infection is coughing, sneezing, talking and uncontrolled movement within and outside the hospital. A hospital monitoring study in Ghana found that $91 \%$ of swabs were positive for various micro-organisms such as coagulasenegative staphylococcus, Bacillus sp, E.coli, Klebsiella sp., and Pseudomonas aeruginosa. ${ }^{11,12}$ In another study by poline et al, Staphylococcus aureus, Pseudomonas aeruginosa, Klebsiella pneumonia, Enterococcus species are responsible for nosocomial infections.

These organisms are constantly present in the hospital, whereas, in our study, 53.8\% (35) were isolated as Pseudomonas aeruginosa, $18 \%(12)$ isolates were identified as Klebsiella pneumonia, $16 \%$ (11) isolates were MethicillinSensitive Staphylococcus aureus (MSSA), 6\% (4) were Coagulase-Negative Staphylococcus (CONS), 3\% (2) were Methicillin-Resistant Staphylococcus aureus (MRSA) and $1.6 \%$ (1) were identified as Enterococcus species.

Pseudomonas aeruginosa was the predominant microorganism causing HAls. Certain studies show that $34 \%$ of Pseudomonas aeruginosa were multidrug-resistant, and $22 \%$ were sensitive strains compared to our research, which shows $26 \%$ were MDR pseudomonas and $74 \%$ were sensitive strains. ${ }^{12}$ Staphylococcus aureus was identified as the predominant cause of HAls. It spreads through the hands of health care personnel. Different studies from South Africa and Switzerland have reported the appearance

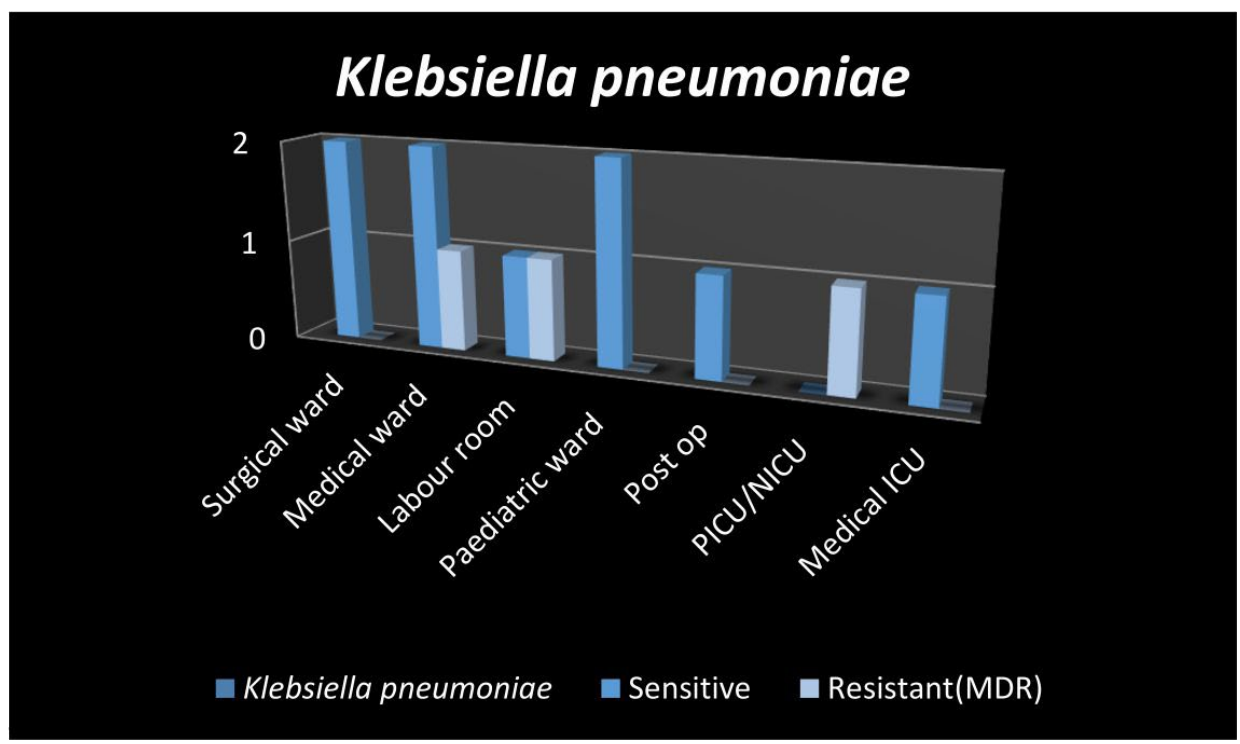

Fig 3. The sensitive strains and resistant strains of Klebsiella pneumonia. 
of resistance to antibacterial agents by Klebsiella and Enterobacter strains has caused Healthcareassociated infections related to the gram-negative bacteria. ${ }^{13,14}$ In our study, 12 (18\%) were identified as Klebsiella pneumonia causing the nosocomial attacks. Among twelve isolates, nine were sensitive to drugs like amoxicillin-clavulanic acid, cefotaxime, ceftrioxzone, ceftazidime, imipenem, amikacin, gentamicin, and cotrimoxazole and three isolates were resistant to all the drugs.

In the study done by Marisa et al., 25\% of isolates in the labor room were Staphylococcus aureus. In our study, $16 \%$ (11) isolates were Methicillin-Sensitive Staphylococcus aureus (MSSA), 6\% (4) were Coagulase-Negative Staphylococcus (CONS), 3\% (2) were MethicillinResistant Staphylococcus aureus (MRSA), and $1.6 \%$ (1) was identified as Enterococcus species ${ }^{15}$. A study done in 1992 by Omori et al. showed the presence of MRSA in environmental samples collected by wiping of floors. ${ }^{16}$ A further study in China found that $60 \%$ of MRSA isolates came from environmental samples, while in our study only $3 \%$ were identified as MRSA.

\section{CONCLUSION}

The prevention and control measures of all nosocomial infections are based on minimizing microorganisms' transmission among the health care personnel and the patients. Also, the prevalence of bacterial sensitivity to antimicrobials forms the basis for designing antibacterial therapy's practical recommendations and for more rational use of antimicrobials. Many measures are taken to control and prevent HAls, such as hand hygiene technique, wearing personal protective equipment, proper donning and doffing of PPE kits used, aseptic non-touch techniques especially in neonatal and paediatric ICUs, and maintaining good respiratory hygiene. Any act of carelessness in the hospital premises like improper maintenance of the wards, poor hand hygiene can lead to nosocomial outbreaks. Proper display of all Information, Education, and Communication (IEC) materials at appropriate places play a significant role in preventing nosocomial infections. Audiovisual aids and training to the staff play utmost importance in preventing the spread of Hospital Acquired
Infections. These all can reduce the occurrence and outbreak of nosocomial infection and reduce the health care cost.

\section{ACKNOWLEDGMENTS}

None.

\section{CONFLICT OF INTEREST}

The authors declare that there is no conflict of interest.

\section{AUTHORS' CONTRIBUTION}

All authors listed have made a substantial, direct and intellectual contribution to the work, and approved it for publication.

\section{FUNDING}

None.

\section{DATA AVAILABILITY}

All datasets generated or analyzed during this study are included in the manuscript

\section{ETHICS STATEMENT}

Not applicable.

\section{REFERENCES}

1. Gravel D, Taylor G, Ofner M, et al. Canadian Nosocomial Infection Surveillance Program. Point prevalence survey for healthcare-associated infections within Canadian adult acute-care hospitals. Journal of Hospital Infection. 2007;66(3):243-248. doi: 10.1016/j. jhin.2007.04.008

2. Dancer SJ. Importance of the environment in Methicillin-resistant Staphylococcus aureus acquisition: the case for hospital cleaning. Lancet Infect Dis. 2008;8(2):101-113. doi: 10.1016/S14733099(07)70241-4

3. Stone PW, Braccia D, Larson E. Systematic review of economic analyses of health care-associated infections. Am J Infect Control. 2005;33(9):501-509. doi: 10.1016/j.ajic.2005.04.246

4. Sydnor ER, Perl TM. Hospital epidemiology and infection control in acute-care settings. Clin Microbiol Rev. 2011;24(1):141-173. doi: 10.1128/CMR.00027-10

5. Allegranzi B, Pittet D. Preventing infections acquired during health-care delivery. Lancet. 2008;372(9651):1719-1720. doi: 10.1016/S01406736(08)61715-8

6. Magill SS, Edwards JR, Bamberg W, Beldavs ZG, Dumyati G, Kainer MA, Lynfield R, Maloney M, McAllister-Hollod L, Nadle J, Ray SM. Multistate point-prevalence survey of health care-associated infections. New England Journal of Medicine. 2014 Mar 27;370(13):1198-208.

7. Wagh A, Sinha A. Prevention of healthcare-associated 
infections in paediatric intensive care unit. Child's Nerv Syst. 2018;34(10):1865-1870. doi: 10.1007/s00381018-3909-4

8. Dilnessa T, Bitew A. Prevalence and antimicrobial susceptibility pattern of methicillin resistant Staphylococcus aureus isolated from clinical samples at Yekatit 12 Hospital Medical College, Addis Ababa, Ethiopia. BMC Infect Dis. 2016;16(1):398. doi: 10.1186/ s12879-016-1742-5

9. Kramer A, Schwebke I, Kampf G. How long do nosocomial pathogens persist on inanimate surfaces? A systematic review. BMC Infect Dis. 2006;6(1):130. doi: 10.1186/1471-2334-6-130

10. Ogwang $M$, Pramatti D, Molteni T, et al. Prevalence of hospital-associated infections can be decreased effectively In developing countries. J Hosp Infect. 2013;84(2):138-142. doi: 10.1016/j.jhin.2013.02.016

11. Newman MJ. Neonatal intensive care unit: reservoirs of Nosocomial pathogens. West Afr J Med. 2002;21(4):310-312. doi: 10.4314/wajm.v21i4.28007
12. Polin RA, Saiman L. Nosocomial infections in the Neonatal Intensive Care. NeoReviews. 2003;4(3):e81-e89. doi: https://doi.org/10.1542/neo.4-3-e81

13. Cotton $\mathrm{MF}$, Wasserman $\mathrm{E}$, Pieper $\mathrm{CH}$, et al.. Invasive disease due to extended spectrum beta-lactamaseproducing Klebsiella pneumoniae in a neonatal unit: the possible role of cockroaches. J Hosp Infect. 2000;44(1):13-17. doi: 10.1053/jhin.1999.0650

14. Harbarth S, Sudre $P$, Dharan S, Cadenas M, Pittet D. Outbreak of Enterobacter cloacae related to understaffing, overcrowding, and poor hygiene practices. Infect Control Hosp Epidemiology. 1999;20(9):598-603. doi: 10.1086/501677

15. Mussi-Pinhata MM, Nascimento SD. Neonatal nosocomial infections. J Pediatric (Rio J). 2001;77(Supl.1):s81-s96. doi: 10.2223/jped.222

16. Omori A, Takahashi A, Watanabe K, et al. Isolation of MRSA from inpatients, staff and environment in the hospital. Kansenshogaku Zasshi. 1992;66(10):1396-1403. doi: 10.11150/ kansenshogakuzasshi1970.66.1396 